\title{
Paeoniflorin inhibits human pancreatic cancer cell apoptosis via suppression of MMP-9 and ERK signaling
}

\author{
NANMU YANG, HONG CUI, FENG HAN, LING ZHANG, TAO HUANG, YI ZHOU and JINXUE ZHOU \\ Department of Hepatopancreatobiliary Surgery, The Affiliated Tumor Hospital of Zhengzhou University, \\ Henan Cancer Hospital, Zhengzhou, Henan 450008, P.R. China
}

Received December 9, 2014; Accepted January 22, 2016

DOI: $10.3892 / 01.2016 .4761$

\begin{abstract}
Paeoniflorin exhibits anticancer, anti-inflammatory and antioxidation effects, as well as specific pharmacological effects on smooth muscle and the immune, cardiovascular and central nervous systems. The present study aimed to investigate the anticancer effects of paeoniflorin on pancreatic cancer cells and to elucidate the mechanisms by which these effects occur. In the present study, 3-(4,5-dimethylthiazol-2-yl)-2,5-diphenyltetrazolium bromide and lactate dehydrogenase assays were performed to assess cell viability and cell cytotoxicity of BXPC-3 human pancreatic cancer cells, respectively. Cellular apoptosis and caspase-3/9 activities were analyzed using an Annexin V-fluorescein isothiocyanate/propidium iodide Apoptosis Detection kit, a DAPI staining assay and colorimetric kits, respectively. Matrix metalloproteinase-9 (MMP-9) and extracellular signal-regulated kinases (ERK) protein expression in BXPC-3 cells were also investigated using gelatin zymography assays and western blot analysis, respectively. In the present study, paeoniflorin was found to inhibit the cell viability and increase cell cytotoxicity of BXPC-3 cells in a dose- and time-dependent manner. In addition, cellular apoptosis, as well as caspase- 3 and -9 activity of BXPC- 3 cells was increased following paeoniflorin treatment. Notably, paeoniflorin reduced MMP-9 and ERK protein expression in BXPC-3 cells. These results indicate that paeoniflorin exhibits a potential anticancer effect by enhancing human pancreatic cancer cell apoptosis via the suppression of MMP-9 and ERK signaling.
\end{abstract}

\section{Introduction}

Although pancreatic cancer is not a common human malignancy, the mortality rate is extremely high at $\sim 100 \%$, which is the leading cause of cancer-related mortality (1). In recent

Correspondence to: Mr. Feng Han, Department of Hepatopancreatobiliary Surgery, The Affiliated Tumor Hospital of Zhengzhou University, Henan Cancer Hospital, 127 East Road, Zhengzhou, Henan 450008, P.R. China

E-mail: fenghanmr@163.com

Key words: paeoniflorin, pancreatic cancer, MMP-9, ERK years, economic development, improvements in standards of living and aging populations have led to an increase in the incidence of pancreatic cancer (2).

Matrix metalloproteinase-9 (MMP-9) is a protease closely associated with tumor invasion and metastasis, as well as the degradation of the basement membrane during vascular formation (3). Angiogenesis is closely associated with tumor growth and metastasis, and microvessel density is an objective indicator that reflects the angiogenesis of tumor tissues. Peng et al showed that increased MMP-9 expression induced by pancreatic cancer cells mediates natural killer cell dysfunction (4). Guo et al reported that ginsenoside Rg3 inhibited pancreatic cancer via the downregulation of MMP9/MMP2 expression (5).

Extracellular signal-regulated kinases (ERK) are a subfamily of the mitogen-activated protein kinase (MAPK) family, that may be activated by a number of cytokines and growth factors to mediate cell proliferation, differentiation and signal transduction (6). ERK1 and ERK2 are two important family members, and the signal transduction pathways in which they are involved are closely associated with the occurrence and development of tumors (7). Furthermore, Tyagi et al (8) indicated that P-21 activated kinase 4 promotes the proliferation and survival of pancreatic cancer cells via the ERK pathway. In addition, Li et al (9) reported that hyperglycemia regulates the thioredoxin-interacting protein/ thioredoxin/reactive oxygen species axis of pancreatic cancer via the p38 MAPK and ERK pathways. Zheng et al (10) reported that gemcitabine inhibited tumour growth and promoted apoptosis of pancreatic cancer via upregulation of pERK1/2 levels. Showalter et al (11) showed that naturally occurring vitamin $\mathrm{K}$ inhibits pancreatic cancer cell survival via the suppression of ERK phosphorylation.

Paeoniflorin was first isolated from the Ranunculaceae plant, peony in 1963 (12). Previous studies have shown that paeoniflorin exhibits antispasmodic, analgesic, antipyretic, anti-inflammatory, anti-ulcer, anti-oxidation, anti-coagulation and regulatory effects; however, the mechanism remains unclear, and a number of receptors and ion channels have been implicated as major targets of paeoniflorin's pharmacological effects (13-16). Paeoniflorin inhibited human pancreatic cancer apoptosis, and the mechanisms are considered to be involved with MMP-9 expression and ERK signaling pathways. Thus, the aim of the present study was to investigate the 
anticancer effects and molecular mechanisms of paeoniflorin on human pancreatic cancer cell apoptosis.

\section{Materials and methods}

Reagents. The chemical structure of paeoniflorin (purity $\geq 98 \%$; Sigma-Aldrich, St. Louis, MO, USA) is shown in Fig. 1. Gibco Dulbecco's modified eagle medium (DMEM) and fetal calf serum (FBS) were obtained from Thermo Fisher Scientific (Waltham, MA, USA). 3-(4,5-dimethylthiazol-2-yl)-2,5-diphenyltetrazolium bromide (MTT) was obtained from Sangon Biotech Co., Ltd., (Shanghai, China). The Annexin V-fluorescein isothiocyanate (FITC)/ propidium iodide (PI) Apoptosis Detection kit and BCA protein assay kit were obtained from Sigma-Aldrich. Caspase-3 and caspase-9 Activities Assay Kits were purchased from Nanjing KeyGen Biotech Co., Ltd. (Nanjing, China).

Cell line and culture conditions. The BXPC-3 human pancreatic cancer cell line was obtained from the Shanghai Institute of Cell Biology, Chinese Academy of Sciences (Shanghai, China). BXPC-3 cells were cultured with DMEM supplemented with $10 \% \mathrm{FBS}, 100 \mathrm{U} / \mathrm{ml}$ penicillin and $100 \mathrm{U} / \mathrm{ml}$ streptomycin in a humidified chamber at $37^{\circ} \mathrm{C}$ in $5 \% \mathrm{CO}_{2}$. The culture medium was replaced every 2-3 days with fresh complete medium (DMEM containing 10\% FBS with $100 \mathrm{U} / \mathrm{ml}$ penicillin and $100 \mathrm{U} / \mathrm{ml}$ streptomycin).

Cell viability assay. BXPC-3 cells ( $5 \times 10^{4}$ cells/well) were seeded in 96-well plates and cultured with DMEM in a humidified chamber at $37^{\circ} \mathrm{C}$ in $5 \% \mathrm{CO}_{2}$ for $24 \mathrm{~h}$. Next, BXPC-3 cells were cultured with $0,6.25,12.5$ and $25 \mu \mathrm{M}$ paeoniflorin for 0, 24, 48 and $72 \mathrm{~h}$, and cell viability was determined by MTT assay. A total of $20 \mu \mathrm{l} \mathrm{MTT} \mathrm{(5} \mathrm{mg/l;} \mathrm{Sangon} \mathrm{Biotech} \mathrm{Co.,} \mathrm{Ltd.)}$ was added to each well, and the plates were incubated for $4 \mathrm{~h}$ in a humidified chamber at $37^{\circ} \mathrm{C}$ in $5 \% \mathrm{CO}_{2}$. The medium was discarded and $150 \mu \mathrm{l}$ dimethyl sulfoxide was added to each well and agitated for $20 \mathrm{~min}$ at room temperature. Cell viability was determined at a wavelength of $490 \mathrm{~nm}$ using a multi-well spectrophotometer (XL-818; Bio-Tek, Winooksi, VT, USA).

Lactate dehydrogenase ( $L D H)$ assay. BXPC-3 cells $\left(5 \times 10^{4}\right.$ cells/well) were seeded in 96 -well plates and cultured with DMEM in a humidified chamber at $37^{\circ} \mathrm{C}$ in $5 \% \mathrm{CO}_{2}$. BXPC-3 cells were then treated with $0,6.25,12.5$ and $25 \mu \mathrm{M}$ paeoniflorin for $0,24,48$ and $72 \mathrm{~h}$, and cell cytotoxicity was analyzed by LDH assay. A total of $100 \mu \mathrm{l} \mathrm{LDH}$ solution was added to each well and incubated at room temperature for $30 \mathrm{~min}$. The absorbance was quantified at a wavelength of $490 \mathrm{~nm}$ using a multi-well spectrophotometer (XL-818; Bio-Tek).

Flow cytometry. BXPC-3 cells $\left(1 \times 10^{6}\right.$ cells/ well) were seeded in 6-well plates and cultured with DMEM in a humidified chamber at $37^{\circ} \mathrm{C}$ in $5 \% \mathrm{CO}_{2}$. BXPC-3 cells were then treated with $0,6.25,12.5$ and $25 \mu \mathrm{M}$ paeoniflorin for $48 \mathrm{~h}$. Cellular apoptotic rates of the BXPC-3 cells was determined using an Annexin V-FITC/PI Apoptosis Detection kit (Sigma-Aldrich). Briefly, BXPC-3 cells were washed twice (5 min each time) with ice-cold PBS and stained with $10 \mu \mathrm{l} \mathrm{V-FITC} \mathrm{at} \mathrm{room}$ temperature for $30 \mathrm{~min}$ in darkness. Next, $5 \mu \mathrm{l}$ PI was added to the cells and incubated at room temperature for $10 \mathrm{~min}$ in darkness. Cellular apoptosis was analyzed by flow cytometry (EPICS ${ }^{\circledR}$ ALTRA $^{\mathrm{TM}}$; Olympus Corporation, Tokyo, Japan).

DAPI staining assay. BXPC-3 cells $\left(1 \times 10^{6}\right.$ cells/per well) were seeded in 6-well plates and cultured with DMEM in a humidified chamber at $37^{\circ} \mathrm{C}$ in $5 \% \mathrm{CO}_{2}$. BXPC-3 cells were treated with $0,6.25,12.5$ and $25 \mu \mathrm{M}$ paeoniflorin for $48 \mathrm{~h}$, and the apoptotic rate of BXPC-3 cells was determined using a DAPI staining assay. BXPC-3 cells were washed twice with ice-cold PBS, fixed in $4 \%$ paraformaldehyde (Sinopharm Chemical Reagent Co., Ltd., Shanghai, China), permeabilized with $0.1 \%$ Triton $\mathrm{X}-100$ and stained with $2 \mu \mathrm{g} / \mathrm{ml}$ DAPI (Beyotime Institute of Biotechnology, Haimen, China) for 10 min. BXPC-3 cells were then observed under a fluorescence microscope (D810; Nikon Corporation, Tokyo, Japan).

Caspase-3 and -9 colorimetric protease assay. BXPC-3 cells (1x10 $10^{6}$ cells/well) were seeded in 6-well plates and cultured with DMEM in a humidified chamber at $37^{\circ} \mathrm{C}$ in $5 \% \mathrm{CO}_{2}$. BXPC-3 cells were treated with $0,6.25,12.5$ and $25 \mu \mathrm{M}$ paeoniflorin for $48 \mathrm{~h}$, and caspase- 3 and -9 activity was determined using a colorimetric protease assay. In accordance with the manufacturer's protocol (Nanjing KeyGen Biotech Co., Ltd.), BXPC-3 cells lysates were prepared in cell lysis buffer for $30 \mathrm{~min}$ on ice and centrifuged at $12,000 \mathrm{x}$ g for $15 \mathrm{~min}$ at $4^{\circ} \mathrm{C}$. Supernate was collected and the protein concentrations were determined using a Pierce BCA Protein Assay Kit (Thermo Fisher Scientific, Inc., Waltham, MA, USA). Equal amounts of protein (40 ng) were mixed with reaction buffer (Ac-DEVD-pNA for caspase-3, Ac-LEHD-pNA for caspase-9) and incubated at $37^{\circ} \mathrm{C}$ for $2 \mathrm{~h}$ in the dark. Caspase-3 and -9 activity was then measured using a microplate reader (ELX800; Bio-Tek) at an absorbance of $405 \mathrm{~nm}$.

Gelatin zymography assays of MMP-9 activity. BXPC-3 cells $\left(1 \times 10^{6}\right.$ cells/well) were seeded in 6-well plates and cultured with DMEM in a humidified chamber at $37^{\circ} \mathrm{C}$ in $5 \% \mathrm{CO}_{2}$. BXPC-3 cells were treated with $0,6.25,12.5$ and $25 \mu \mathrm{M}$ paeoniflorin for $48 \mathrm{~h}$, and the MMP-9 activity of BXPC-3 cells was determined by gelatin zymography assays. Equal volumes of sample $(40 \mu \mathrm{l})$ were mixed with sodium dodecyl sulfate (SDS) sample buffer (Tiandz Inc., Beijing, China). The miscible liquids were subjected to $10 \%$ SDS-PAGE gels polymerized with $1 \mathrm{mg} / \mathrm{ml}$ gelatin (Tiandz Inc.). The gel was then washed three times for $20 \mathrm{~min}$ at room temperature in $2.5 \%$ Triton X-100 to remove SDS following electrophoresis. Next, the gel was incubated in radioimmunoprecipitation buffer (Nanjing KeyGen Biotech Co., Ltd.) at $37^{\circ} \mathrm{C}$ for $12 \mathrm{~h}$. The gel was stained with $0.05 \%$ Coomassie brilliant blue stain R-250 (Beyotime Institute of Biotechnology) was used to stain followed by destaining with $7 \%$ acetic acid (Sinopharm Chemical Reagent Co., Ltd.).

Western blot analysis of ERK protein expression. BXPC-3 cells $\left(1 \times 10^{6}\right.$ cells/well) were seeded in 6 -well plates and cultured with DMEM in a humidified chamber at $37^{\circ} \mathrm{C}$ in $5 \% \mathrm{CO}_{2}$. BXPC-3 cells were treated with $0,6.25,12.5$ and 


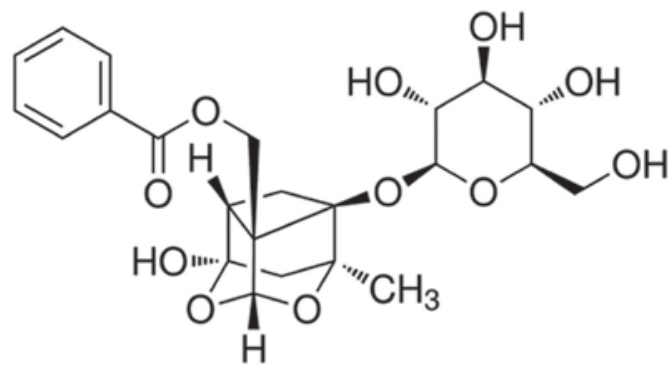

Figure 1. Chemical structure of paeoniflorin.

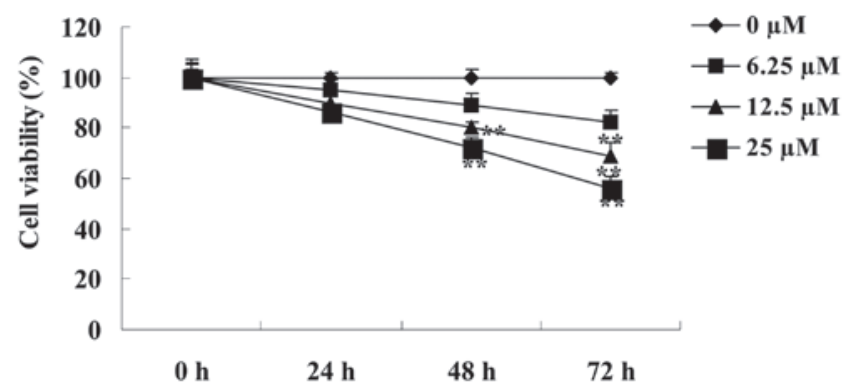

Figure 2. Effect of $0,6.25,12.5$ and $25 \mu \mathrm{M}$ paeoniflorin treatment on cell viability at various time points. ${ }^{* *} \mathrm{P}<0.01$ vs. $0 \mu \mathrm{M}$ paeoniflorin treatment group.

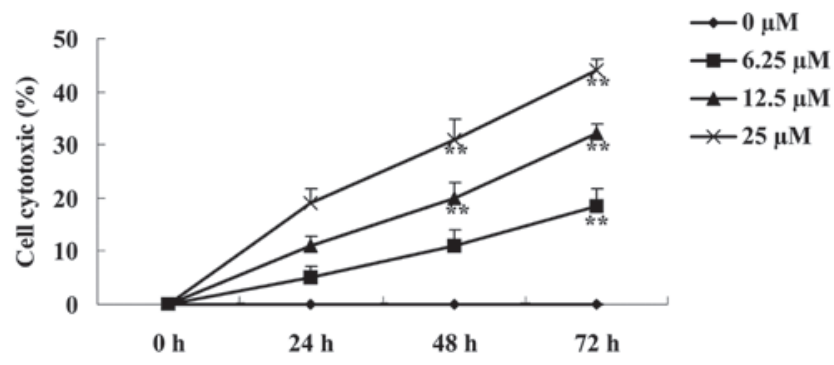

Figure 3. Effect of $0,6.25,12.5$ and 25 paeoniflorin treatment on cell cytotoxicity at various time points. ${ }^{* *} \mathrm{P}<0.01$ vs. $0 \mu \mathrm{M}$ paeoniflorin treatment group.

$25 \mu \mathrm{M}$ paeoniflorin for $48 \mathrm{~h}$, and ERK protein expression was determined by western blot analysis. Briefly, the cells were washed with cold PBS and incubated with ice-cold lysis buffer for $30 \mathrm{~min}$ on ice. BXPC-3 cell liquid was collected and centrifuged at $12,000 \mathrm{x}$ g for $15 \mathrm{~min}$ at $4^{\circ} \mathrm{C}$. The protein concentrations were determined using a Pierce BCA Protein Assay Kit (Thermo Fisher Scientific, Inc.). Equal protein was separated using $12 \%$ SDS-polyacrylamide gels with Coomassie Brilliant Blue, and transferred to a polyvinylidene fluoride membrane (0.22 mm; EMD Millipore, Bedford, MA, USA). The blotted membranes were then blocked with Tris-buffered saline containing 5\% non-fat milk to block nonspecific binding sites. The transferred membrane was incubated with polyclonal rabbit anti-ERK (1:1,500; sc-292838; Santa Cruz Biotechnology, Dallas, TX, USA) and polyclonal rabbit anti- $\beta$-actin (1:500; sc-130657; Sangon Biotech Co., Ltd.,) antibodies overnight at $4^{\circ} \mathrm{C}$. The membrane was washed with TBST and incubated with rabbit anti-mouse immunoglobulin $\mathrm{G}$ horseradish peroxidase-conjugated antibody (1:5,000; A1949, Sigma-Aldrich) for $1 \mathrm{~h}$. Then the proteins were detected using enhanced chemiluminescence using an ECL Advanced Western Blot detection Kit (Tiangen Biotech Co., Ltd., Beijing, China). and then the proteins were detected using enhanced chemiluminescence using an ECL Advanced Western Blot detection Kit (Tiangen Biotech Co., Ltd., Beijing, China).

Statistical analysis. All statistical analyses were performed using SPSS 19.0 statistical software (SPSS Inc., Chicago, IL, USA) and representative of at least three independent experiments. Analysis of variance was performed to compare multiple data. Data are expressed as the mean \pm standard deviation of at least three independent experiments. $\mathrm{P}<0.05$ was considered to indicate a statistically significant difference.

\section{Results}

Effect of paeoniflorin on cell viability. The effect of paeoniflorin on the cell viability of BXPC-3 cells was determined using MTT assays. As shown in Fig. 2, treatment with 6.25, 12.5 and $25 \mu \mathrm{M}$ paeoniflorin decreased cell viability in a dose- and time-dependent manner. Following treatment with $6.25 \mu \mathrm{M}$ paeoniflorin for $72 \mathrm{~h}$, and treatment with 12.5 and $25 \mu \mathrm{M}$ paeoniflorin for 48 and $72 \mathrm{~h}$, the cell viability of BXPC-3 cells was significantly reduced when compared with the $0 \mu \mathrm{M}$ paeoniflorin-treatment group ( $\mathrm{P}<0.01$; Fig. 2). Based on these results, a dose of $12.5 \mu \mathrm{M}$ paeoniflorin and a treatment duration of $48 \mathrm{~h}$ were selected for further study.

Effect of paeoniflorin on cell cytotoxicity. The effect of paeoniflorin on BXPC-3 cell cytotoxicity was investigated using the LDH release assay. As shown in Fig. 3, cell cytotoxicity of BXPC-3 cells increased following treatment with 6.25 , 12.5 and $25 \mu \mathrm{M}$ paeoniflorin, in a dose- and time-dependent manner. Notably, in cells treated with $6.25 \mu \mathrm{M}$ paeoniflorin for $72 \mathrm{~h}$, and 12.5 and $25 \mu \mathrm{M}$ paeoniflorin for 48 and $72 \mathrm{~h}$, cell cytotoxicity of BXPC-3 cells was significantly increased when compared with the $0 \mu \mathrm{M}$ paeoniflorin treatment group $(\mathrm{P}<0.01$; Fig. 3).

Effect of paeoniflorin on cellular apoptosis. To determine the effect of paeoniflorin on cellular apoptosis of BXPC-3 cells, the levels of cellular apoptosis were analyzed by Annexin V-FITC/PI apoptosis and DAPI staining assays. Treatment with $6.25,12,5$ and $25 \mu \mathrm{M}$ paeoniflorin for $48 \mathrm{~h}$ induced a concentration-dependent increase in cellular apoptosis of BXPC-3 cells (Fig. 4A-B). As shown in Fig. 4A, treatment with 12.5 and $25 \mu \mathrm{M}$ paeoniflorin resulted in a significant increase in cellular apoptosis of BXPC-3 cells at $48 \mathrm{~h}$ when compared with that of the $0 \mu \mathrm{M}$ paeoniflorin-treatment group $(\mathrm{P}<0.01$; Fig. 4A). In addition, DAPI staining revealed that cellular apoptosis of BXPC-3 cells was increased in the 6.25, 12.5 and $25 \mu \mathrm{M}$ paeoniflorin treatment groups at $48 \mathrm{~h}$ when compared with the $0 \mu \mathrm{M}$ paeoniflorin treatment group (Fig. 4B).

Effect of paeoniflorin on caspase-3 and -9 activity. The effect of paeoniflorin treatment on caspase- 3 and caspase- 9 activity of BXPC-3 cells was also assessed. Caspase- 3 and caspase- 9 


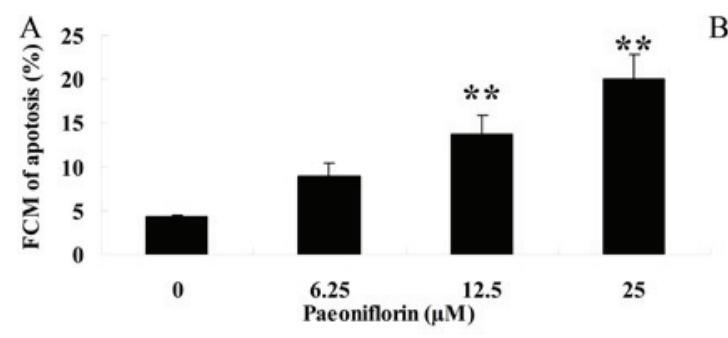

B

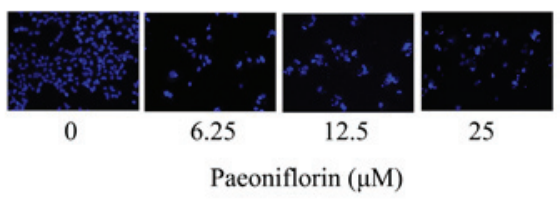

Figure 4. Effect of $0,6.25,12.5$ and $25 \mu \mathrm{M}$ paeoniflorin treatment on cellular apoptosis of BXPC-3 cells, following treatment for $48 \mathrm{~h}$. (A) Quantification of flow cytometry results. ${ }^{* *} \mathrm{P}<0.01$ vs. $0 \mu \mathrm{M}$ paeoniflorin treatment group. (B) DAPI staining assay.
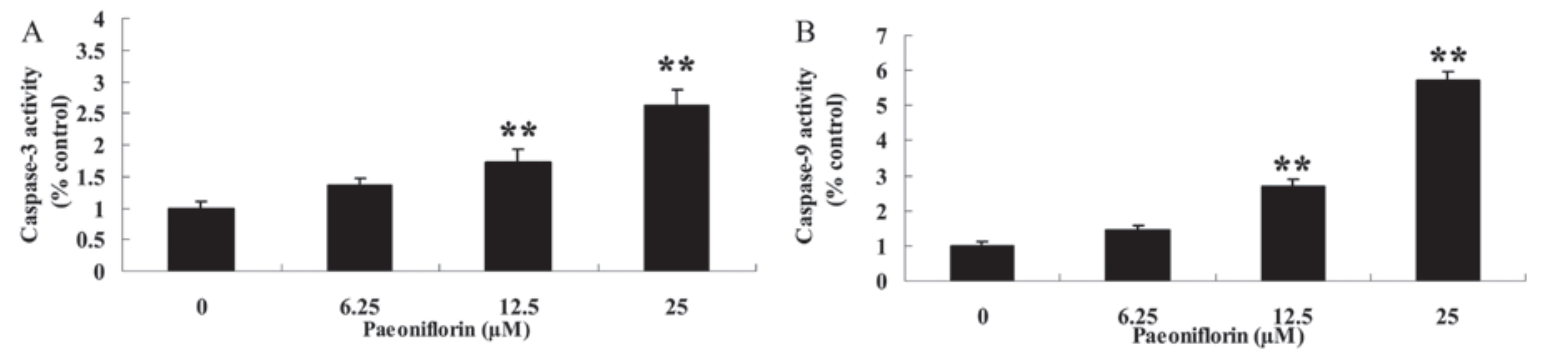

Figure 5. Effect of treatment with $0,6.25,12.5$ and $25 \mu \mathrm{M}$ paeoniflorin for $48 \mathrm{~h}$ on (A) caspase-3 and (B) -9 activity of BXPC-3 cells. ${ }^{* *} \mathrm{P}<0.01$ vs. $0 \mu \mathrm{M}$ paeoniflorin treatment group.
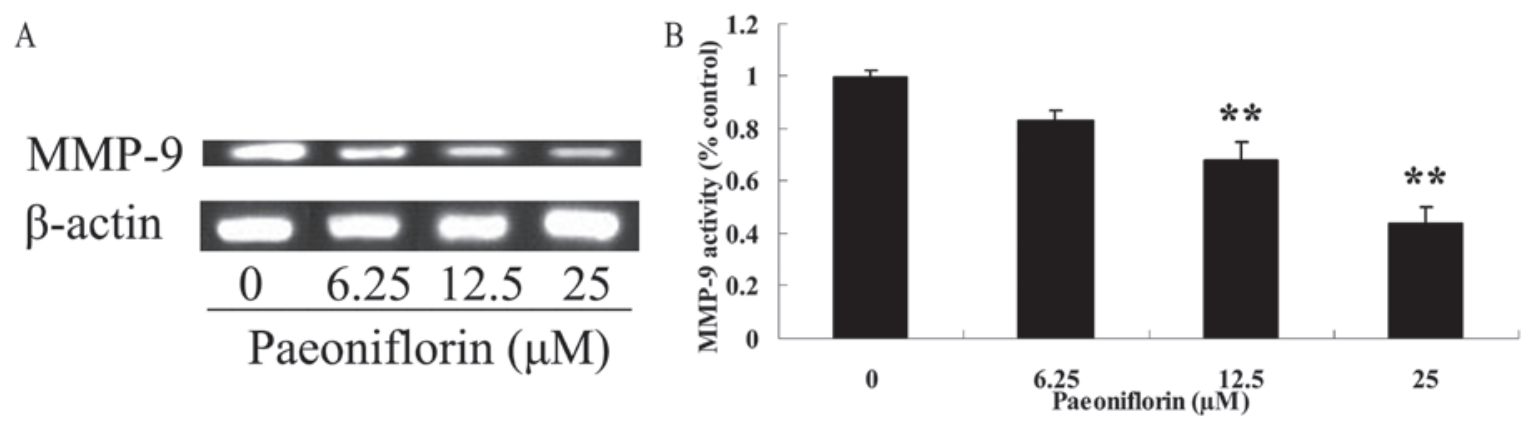

Figure 6. Effect of treatment with 0, 6.25, 12.5 and $25 \mu \mathrm{M}$ paeoniflorin for $48 \mathrm{~h}$ on MMP-9 activity. (A) The effects of genistein on MMP-9 activity of BXPC-3 cells by gelatin zymography assays and (B) quantification of MMP-9 activity in BXPC-3 cells. ${ }^{* *} \mathrm{P}<0.01 \mathrm{vs.} 0 \mu \mathrm{M}$ paeoniflorin treatment group. MMP-9, matrix metalloproteinase 9 .

A

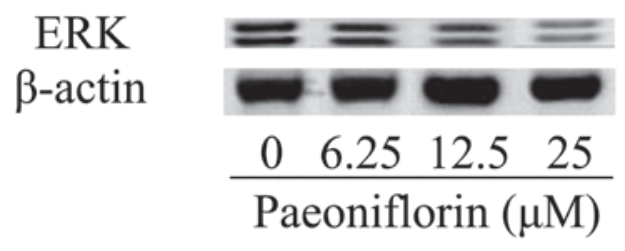

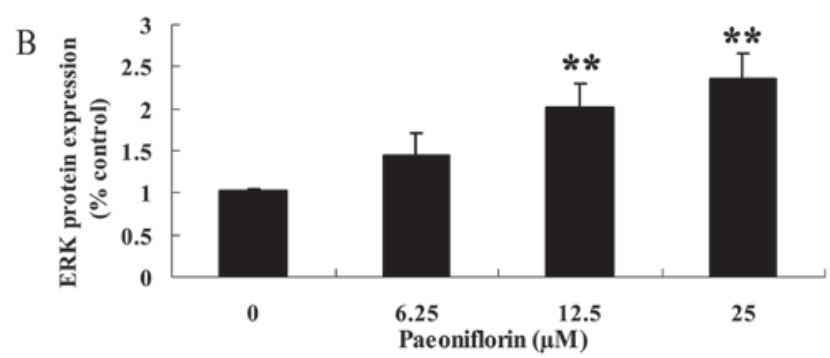

Figure 7. Effect of treatment with $0,6.25,12.5$ and $25 \mu \mathrm{M}$ paeoniflorin for $48 \mathrm{~h}$ on ERK protein expression. (A) Western blot analysis and (B) quantification of ERK protein expression of BXPC-3 cells. ${ }^{* *} \mathrm{P}<0.01$ vs. $0 \mu \mathrm{M}$ paeoniflorin treatment group. ERK, extracellular signal-regulated kinase.

activity of BXPC-3 cells was significantly increased following $48 \mathrm{~h}$ treatment with 12.5 and $25 \mu \mathrm{M}$ paeoniflorin when compared with that of $0 \mu \mathrm{M}$ paeoniflorin $(\mathrm{P}<0.01$; Fig. 5A and $\mathrm{B})$. These results indicate that paeoniflorin may promote cellular apoptosis of BXPC-3 cells.
Effect of paeoniflorin on MMP-9 activity. To investigate the effect of paeoniflorin on BXPC-3 cells, MMP-9 activity of BXPC-3 cells was evaluated by gelatin zymography assays. The MMP-9 activity of BXPC-3 cells was significantly reduced by treatment with 12.5 and $25 \mu \mathrm{M}$ paeoniflorin for 
$48 \mathrm{~h}$ when compared with that of the $0 \mu \mathrm{M}$ paeoniflorin treatment group $(\mathrm{P}<0.01$; Fig. $6 \mathrm{~A}$ and $\mathrm{B})$.

Effect of paeoniflorin on ERK protein expression. ERK protein expression of BXPC-3 cells was analyzed using western blot analysis. The ERK protein expression of BXPC-3 cells was significantly increased following 12.5 and $25 \mu \mathrm{M}$ paeoniflorin treatment for $48 \mathrm{~h}$, when compared with that of the $0 \mu \mathrm{M}$ paeoniflorin treatment group $(\mathrm{P}<0.01$; Fig. 7A and B).

\section{Discussion}

The early symptoms of high-grade malignant pancreatic tumors are not clear, and thus the majority of tumors are identified at an advanced stage, yielding a low surgical resection rate (17). As a result of recent changes in human behavior and diet, the worldwide incidence of pancreatic cancer has been increasing annually (18). Pancreatic cancer results from the interaction between genetic and environmental factors, which may be associated with genetic susceptibility as a result of genetic mutation, genetic polymorphism and epigenetic factors. Recently, certain risk factors which are associated with pancreatic cancer, including smoking, obesity, alcohol consumption, chronic pancreatitis and diabetes, have increasingly gained attention (19). In the current study, paeoniflorin was found to decrease cell viability and increase cell cytotoxicity of BXPC-3 cells in a dose- and time-dependent manner. In addition, levels of BXPC-3 cellular apoptosis were increased following paeoniflorin treatment. Notably, the activity of caspase-3 and -9 in BXPC-3 cells was increased by paeoniflorin treatment. In a previous study, Lu et al reported that paeoniflorin inhibited the tumor invasion and metastasis of HepG2 and Bel-7402 human hepatocellular carcinoma cells via the suppression of MMP-9 and ERK (20). Wang et al showed that paeoniflorin inhibited the growth of human colorectal cancer cells via regulation of $\mathrm{p} 53 / 14-3-3 \zeta(21)$.

MMP-9 degrades IV and V collagen and gelatin in the extracellular matrix, which facilitates the growth of tumor blood vessels to mesenchyme and subsequently, the microvessel density of tumor blood vessels increases, leading to continual tumor growth and distant metastasis (22). A previous study showed that the positive expression rate of MMP-9 is 50\% in pancreatic cancer patients, and in cases accompanied by liver metastasis, the positive rate of MMP-9 expression is $66.7 \%$, which indicates MMP-9 may be associated with pancreatic cancer liver metastases (23). The present study demonstrated that MMP-9 activity of human pancreatic cancer BXPC-3 cells was significantly reduced by paeoniflorin treatment, which is in agreement with the results of Lu et al (20) which indicated paeoniflorin inhibited the tumor invasion and metastasis of human hepatocellular cancer cells via suppression of MMP-9 and ERK. Ji et al (24) reported that paeoniflorin suppressed type I collagen synthesis via the inhibition of MMP-1 mRNA expression.

Activated ERK kinase causes cell proliferation and differentiation (25). Jun protein Jen encoded by c-jun belongs to the members of activated protein (AP-1) family, and is a downstream target gene of the MAPK family. Many tumor genes mediate transformed tumorigenic effect by AP-1 family signal transduction pathway, and activated AP-1 family promotes cell transformation and cancerization, participating in all the links including extracellular matrix degradation, abnormality and angiogenesis of metastatic tumor newborn vessels by downstream target gene expression (26). The results of the present study showed that paeoniflorin significantly reduced ERK protein expression levels in human pancreatic cancer BXPC-3 cells. Chen et al showed that paeoniflorin attenuated cerebral infarction via downregulation of mitogen-activated protein kinase kinase (MEK), phosphorylated-MEK and ERK (27). In addition, paeoniflorin inhibited the tumor invasion and metastasis of hepatocellular carcinoma cells via downregulation of MMP-9 and ERK expression (20).

In conclusion, the results of this study suggest that paeoniflorin decreases cell viability and promote cellular apoptosis of human pancreatic cancer BXPC-3 cells. Furthermore, these results indicate that MMP-9 and ERK may significantly contribute to the anticancer effects of paeoniflorin. However, further studies are required to identify additional signaling pathways that are affected by paeoniflorin, which may elucidate to the mechanism of its anticancer effects in pancreatic cancer cells.

\section{References}

1. Nakamura M, Oida Y, Abe Y, Yamazaki H, Mukai M, Matsuyama M, Chijiwa T, Matsumoto $\mathrm{H}$ and Ueyama $\mathrm{Y}$ : Thrombospondin-2 inhibits tumor cell invasion through the modulation of MMP-9 and uPA in pancreatic cancer cells. Mol Med Rep 1: 423-427, 2008.

2. Bakshi H, Sam S, Rozati R, Sultan P, Islam T, Rathore B, Lone Z, Sharma M, Triphati J and Saxena RC: DNA fragmentation and cell cycle arrest: A hallmark of apoptosis induced by crocin from kashmiri saffron in a human pancreatic cancer cell line. Asian Pac J Cancer Prev 11: 675-679, 2010.

3. Klein G, Vellenga E, Fraaije MW, Kamps WA and de Bont ES: The possible role of matrix metalloproteinase (MMP)-2 and MMP-9 in cancer, e.g. acute leukemia. Crit Rev Oncol Hematol 50: 87-100, 2004.

4. Peng YP, Zhang JJ, Liang WB, Tu M, Lu ZP, Wei JS, Jiang KR, Gao WT, Wu JL, Xu ZK, et al: Elevation of MMP-9 and IDO induced by pancreatic cancer cells mediates natural killer cell dysfunction. BMC Cancer 14: 738, 2014.

5. Guo JQ, Zheng QH, Chen H, Chen L, Xu JB, Chen MY, Lu D, Wang ZH, Tong HF and Lin S: Ginsenoside Rg3 inhibition of vasculogenic mimicry in pancreatic cancer through downregulation of VE-cadherin/EphA2/MMP9/MMP2 expression. Int J Oncol 45: 1065-1072, 2014.

6. Xie G, Yang S, Chen A, et al: Electroacupuncture at quchi and zusanli treats cerebral ischemia-reperfusion injury through activation of ERK signaling. Exp Ther Med 5: 1593-1597, 2013.

7. Amsterdam A, Shezen E, Raanan C, Schreiber L, Prus D, Slilat Y, Ben-Arie A and Seger R: Nuclear localization of phosphorylated ERK1 and ERK2 as markers for the progression of ovarian cancer. Int J Oncol 39: 649-656, 2011.

8. Tyagi N, Bhardwaj A, Singh AP, McClellan S, Carter JE and Singh S: $p-21$ activated kinase 4 promotes proliferation and survival of pancreatic cancer cells through AKT- and ERK-dependent activation of NF-kB pathway. Oncotarget 5: 8778-8789, 2014.

9. Li W, Wu Z, Ma Q, et al: Hyperglycemia regulates TXNIP/TRX/ ROS axis via 38 MAPK and ERK pathways in pancreatic cancer. Curr Cancer Drug Targets 14: 348-356, 2014.

10. Zheng C, Jiao X, Jiang Y and Sun S: ERK1/2 activity contributes to gemcitabine resistance in pancreatic cancer cells. J Int Med Res 41: 300-306, 2013.

11. Showalter SL, Wang Z, Costantino CL, et al: Naturally occurring $\mathrm{K}$ vitamins inhibit pancreatic cancer cell survival through a caspase-dependent pathway. J Gastroenterol Hepatol 25: 738-744, 2010. 
12. Qiu F, Zhong X, Mao Q and Huang Z: The antidepressant-like effects of paeoniflorin in mouse models. Exp Ther Med 5: 1113-1116, 2013.

13. Li J,Ji X,Zhang J, Shi G,Zhu X and Wang K: Paeoniflorin attenuates A $325-35$-induced neurotoxicity in PC12 cells by preventing mitochondrial dysfunction. Folia Neuropathol 52: 285-290, 2014.

14. Wang F, Wang CM, Liu JD and Wang YT: Influence of paeoniflorin on intracellular calcium ion concentration in the sphincter of Oddi of hypercholesterolemic rabbits. Genet Mol Res 13: 5001-5010, 2014.

15. Tsuboi H,Hossain K, Akhand AA, Takeda K, Du J, Rifa'i M, Dai Y, Hayakawa A, Suzuki H and Nakashima I: Paeoniflorin induces apoptosis of lymphocytes through a redox-linked mechanism. J Cell Biochem 93: 162-172, 2004.

16. Wang C, Yuan J, Wu HX, Chang Y, Wang QT, Wu YJ, Liu LH and Wei W: Paeoniflorin inhibits inflammatory responses in mice with allergic contact dermatitis by regulating the balance between inflammatory and anti-inflammatory cytokines. Inflamm Res 62: 1035-1044, 2013.

17. Mori-Iwamoto S, Kuramitsu Y, Ryozawa S, Taba K, Fujimoto M, Okita K, Nakamura K and Sakaida I: A proteomic profiling of gemcitabine resistance in pancreatic cancer cell lines. Mol Med Rep 1: 429-434, 2008

18. Taghavi A, Fazeli Z, Vahedi M, Baghestani AR, Zali MR and Pourhoseingholi MA: Pancreatic cancer mortality and misclassification-bayesian analysis. Asian Pac J Cancer Prev 12: 2271-2274, 2011

19. Li S, Sun J, Yang J, Zhang L, Wang L, Wang X and Guo Z: XIAP expression is associated with pancreatic carcinoma outcome. Mol Clin Oncol 1: 305-308, 2013.

20. Lu JT, He W, Song SS and Wei W: Paeoniflorin inhibited the tumor invasion and metastasis in human hepatocellular carcinoma cells. Bratisl Lek Listy 115: 427-433, 2014.
21. Wang H, Zhou H, Wang CX, Li YS, Xie HY, Luo JD and Zhou Y: Paeoniflorin inhibits growth of human colorectal carcinoma HT 29 cells in vitro and in vivo. Food Chem Toxicol 50: 1560-1567, 2012.

22. Zhang Y, Wu JZ, Zhang JY, Xue J, Ma R, Cao HX and Feng J: Detection of circulating vascular endothelial growth factor and matrix metalloproteinase-9 in non-small cell lung cancer using Luminex multiplex technology. Oncol Lett 7: 499-506, 2014.

23. Yuan J, Wu Y and Lu G: $\alpha$-Mangostin suppresses lipopolysaccharide-induced invasion by inhibiting matrix metalloproteinase-2/9 and increasing E-cadherin expression through extracellular signal-regulated kinase signaling in pancreatic cancer cells. Oncol Lett 5: 1958-1964, 2013

24. Ji Y, Wang T, Wei ZF, Lu GX, Jiang SD, Xia YF and Dai Y: Paeoniflorin, the main active constituent of Paeonia lactiflora roots, attenuates bleomycin-induced pulmonary fibrosis in mice by suppressing the synthesis of type I collagen. J Ethnopharmacol 149: 825-832, 2013.

25. Al-Wadei HA, Al-Wadei MH, Ullah MF and Schuller HM: Celecoxib and GABA cooperatively prevent the progression of pancreatic cancer in vitro and in xenograft models of stress-free and stress-exposed mice. PLoS One 7: e43376, 2012.

26. Shen YJ,Zhu XX, Yang X, Jin B, Lu JJ, Ding B, Ding ZS and Chen SH: Cardamonin inhibits angiotensin II-induced vascular smooth muscle cell proliferation and migration by downregulating p38 MAPK, Akt, and ERK phosphorylation. J Nat Med 68: 623-629, 2014.

27. Chen YF, Wu KJ and Wood WG: Paeonia lactiflora extract attenuating cerebral ischemia and arterial intimal hyperplasia is mediated by paeoniflorin via modulation of VSMC migration and Ras/MEK/ERK signaling pathway. Evid Based Complement Alternat Med 2013: 482428, 2013. 